\title{
FTY720 inhibits tubulointerstitial inflammation in albumin overload-induced nephropathy of rats via the Sphk1 pathway
}

\author{
Min XU, Dan LIU, Li-hong DING, Kun-ling MA, Min WU, Lin-li LV, Yi WEN, Hong LIU, Ri-ning TANG, Bi-cheng LIU* \\ Institute of Nephrology, Division of Nephrology, Zhongda Hospital, School of Medicine, Southeast University, Nanjing 210009, China
}

\begin{abstract}
Aim: FTY720, a new immunomodulatory drug with low cytotoxicity, is currently used to treat multiple sclerosis. In this study, we investigated the effects of FTY720 on inflammatory cell infiltration in albumin overload-induced nephropathy of rats.

Methods: Male Wistar rats were subjected to right-side nephrectomy and divided into 3 groups. One week after the surgery, albumin overload (AO) group was treated with BSA ( $5 \mathrm{~g}_{\mathrm{kg}} \mathrm{g}^{-1} \cdot \mathrm{d}^{-1}$, ip) for 9 weeks; AO+FTY720 group was given BSA (5 g:kg $\mathrm{kg}^{-1} \cdot \mathrm{d}^{-1}$, ip) plus FTY720 $\left(0.5 \mathrm{~g}_{\mathrm{kg}} \cdot \mathrm{d}^{-1}\right.$, ip) for 9 weeks; and control group received daily ip injection of equivalent volume of saline. All rats were killed 9 weeks after nephrectomy.

Results: $\mathrm{AO}$ rats exhibited gradually increased urinary protein excretion accompanied by elevated urinary $\mathrm{N}$-acetyl- $\beta$ - 0 -glucosaminidase activity, and both reached their peak values at week 7 . Furthermore, AO significantly increased lymphocytes and monocytes in circulation and the inflammatory cells recruited to tubulointerstitium, and the expression of inflammatory cytokines MCP-1, TNF- $\alpha$ and IL-6, as well as sphingosine 1-phosphate (S1P) receptors S1pr1 and S1pr3, and S1P-synthesizing enzyme sphingosine kinase 1 (Sphk1) in the kidney. Concomitant administration of FTY720 significantly attenuated all the AO-induced pathological changes. Conclusion: FTY720 alleviates tubulointerstitium inflammation in an AO rat model of nephropathy via down-regulation of the Sphk1 pathway.
\end{abstract}

Keywords: FTY720; chronic kidney disease; nephropathy; albumin overload rat model; proteinuria; tubulointerstitium; inflammation; cytokine; Sphk1 pathway

Acta Pharmacologica Sinica (2014) 35: 1537-1545; doi: 10.1038/aps.2014.100; published online Nov 172014

\section{Introduction}

Tubulointerstitial inflammation (TII) is one of the most important pathological features in chronic kidney disease (CKD) which can predict the outcome of CKD. In the past decades, a number of studies have demonstrated that proteinuria is not only a marker of CKD but also a risk factor for CKD to progress to end-stage renal disease ${ }^{[1]}$. Furthermore, a large body of evidence has indicated that increased protein absorption by tubular cells may result in direct tubular toxicity, release of chemokines and cytokines, and increased expression of adhesion molecules ${ }^{[2,3]}$. The net effect is an increased influx of mononuclear inflammatory cells into the tubulointerstitium. There is little doubt that prolonged inflammation has an important connection to the development of fibrosis ${ }^{[4]}$, therefore, reducing proteinuria using an angiotensin convert-

\footnotetext{
* To whom correspondence should be addressed. E-mail liubc64@163.com

Received 2014-05-05 Accepted 2014-09-05
}

ing enzyme inhibitor (ACEI), angiotensin receptor blocker (ARB) or an immunosuppressant has long been regarded as an important strategy for treating proteinuric renal diseases.

The newly developed immunosuppressant drug FTY720, currently used as the first-line oral treatment for multiple sclerosis ${ }^{[5]}$, is a structural analog of sphingosine and has low cytotoxicity. FTY720 is phosphorylated to FTY720-P by SphK2 in vivo. FTY720-P, which shares structural homology with the natural ligand sphingosine 1-phosphate (S1P), functions as a potent "agonist" or "antagonist" for all of the S1P receptors except S1pr2 ${ }^{[6,7]}$. FTY720-induced lymphopenia was shown to be due to the inhibition of lymphocyte recruitment from lymphoid organs in an S1pr1-dependent manner, but FTY720 does not affect the proliferation and activation of lymphocytes ${ }^{[8]}$. Similar to lymphocytes, FTY720 treatment was also demonstrated to restrict NK cell and circulating monocyte recruitment from the spleen and bone marrow. As a result, FTY720 decreased the number of inflammatory cells in the peripheral inflammatory tissues ${ }^{[9]}$. Furthermore, the $\mathrm{S}_{1} \mathrm{P}_{3}$ receptor in 
macrophages was shown to mediate their migration properties and inflammatory potential and to promote their recruitment to inflammation sites and atherosclerotic lesions. FTY720 treatment alleviated this inflammatory response ${ }^{[3]}$.

Previous studies have demonstrated a remarkable protective effect of FTY720 in models of 5/6 nephrectomized renal failure $^{[10]}$, acute renal ischemia-reperfusion injury ${ }^{[11]}$ and diabetic nephropathy ${ }^{[12]}$. However, the precise effect of FTY720 on proteinuria-induced tubulointerstitial inflammation has not been well addressed. In this study, using the albuminoverload (AO) model, we demonstrated that FTY720 attenuated lymphocyte and macrophage infiltration and inflammatory mediator expression. Our data provide further evidence that FTY720 might be a novel therapeutic strategy for treating proteinuric nephropathy.

\section{Materials and methods Animals}

Male Wistar rats (180-200 g, 6 week) were purchased from Beijing Military Academy of Medical Sciences Laboratory Animal Center (Beijing, China) and housed in cages at stable room temperature and humidity under $12 \mathrm{~h}$ light/dark cycles. They were fed standard rat chow and had free access to water. All procedures were performed in accordance with the university guidelines, and all protocols were approved by the Ethics Review Committees for Animal Experimentation of Southeast University (SYXK 2010-0004).

\section{AO (albumin-overload) model and drug treatment}

After 1 week of adaptive feeding, each rat was anesthetized with chloral hydrate solution and underwent a right-side nephrectomy. Then, they were divided into three groups: 1 ) Saline control, daily intraperitoneal injection of saline solution $(0.09 \%$, equivalent volume of BSA); 2 ) AO (albumin overload), intraperitoneal injection of BSA (bovine serum albumin, Roche, Switzerland) 1 week after surgery $\left(5 \mathrm{~g} \cdot \mathrm{kg}^{-1} \cdot \mathrm{d}^{-1}\right)$ for 9 weeks; and 3) AO+FTY720, FTY720 (Fingolimod, Selleck Chemicals, Houston, USA) administration $\left(0.5 \mathrm{mg} \cdot \mathrm{kg}^{-1} \cdot \mathrm{d}^{-1}\right)$ along with BSA. BSA and FTY720 were dissolved in $0.09 \%$ saline.

Nine weeks later, all the rats were killed. Blood samples taken by puncturing the heart, and left kidneys were collected and weighed. The renal tissue was fixed in $10 \%$ buffered formalin and embedded in paraffin for histological evaluation and immunohistochemical analysis, and the remaining tissue was frozen in liquid nitrogen and stored at $-80^{\circ} \mathrm{C}$ until used for Western blotting and PCR analysis.

\section{Proteinuria and blood biochemical analysis}

At week 0, 2, 5, 7, and 9 after nephrectomy, each rat was placed in a metabolic cage for 24-h urine collection, and the urine was frozen at $-80^{\circ} \mathrm{C}$ until measurement. Proteinuria was measured using the Bradford Method, and NAG activity was determined by an Elisa assay kit (Nanjing Jiancheng Bioengineering Institute, Nanjing, China). Blood count was analyzed via a routine blood test. Serum concentrations of creatinine, urea nitrogen, albumin and total protein were determined using automatic analyzers (Hitachi, Tokyo, Japan).

\section{Histological studies}

Paraffin sections $(2 \mu \mathrm{m})$ were stained with periodic acidSchiff (PAS) reagent. The histological parameter of interstitial inflammation was scored on 20 randomly selected cortical areas per sample, and the lesions were graded from 0 to 3 ( 0 , normal; 1 , lesions involving $<25 \%$ of the cortical area; 2 , $25 \%-50 \%$; and $3,>50 \%)^{[13]}$. The score index in each rat was presented as the mean value of all scores obtained.

\section{Immunohistochemical analysis}

Immunohistochemistry was performed on paraffin sections $(2 \mu \mathrm{m})$ with antibodies against CD68 (macrophages), CD3 (T lymphocytes), CD20 (B lymphocytes), sphingosine 1-phophate receptor 1 and 3 (S1pr1 and S1pr3, Santa Cruz, CA, USA), Sphk1 (sphingosine kinase 1, Abcam, Cambridge, England), followed by incubation with appropriate secondary antibodies. Diaminobenzidine was used for visualization of immunoreactivity, followed by hematoxylin for nuclear counterstaining. In each kidney, at least 20 nonoverlapping cortical areas of tubulointerstitial section were analyzed for evaluating the numbers of CD68-, CD3-, and CD20-positive cells. The area containing Sphk1-, S1pr1-, and S1pr3-positive pixels was expressed as percentage of total inflammatory area.

\section{Quantitative real-time RT-PCR}

Total RNA of kidney cortex was isolated using Trizol reagent (Takara, Japan), and cDNA was synthesized using a reverse transcription system kit (Takara, Japan) according to the manufacturer's instructions. The RNA concentration was measured using a Nanodrop. All real-time RT-PCR reactions were performed on ABI PRISM 7300 real-time PCR system (Applied Biosystems, CA, USA) using the SYBR Premix Ex Taq (Takara, Japan) with gene-specific primer sets of rat monocyte chemoattractant protein-1 (MCP-1), tumor necrosis factor-a (TNFa), interleukin-6 (IL-6), interleukin-10 (IL-10), and arginase-1 developed by Invitrogen (Invitrogen Biotechnology, Shanghai, China). Primer sequences are presented in Table 1 . The abundance of gene expression was normalized to that of $\beta$-actin, and results were analyzed using the $2^{-\Delta \mathrm{Ct}}$ method.

\section{Western blotting}

Total proteins from the renal cortex were separated by SDSPAGE and transferred to a nitrocellulose membrane. After blocking with $5 \%$ skim milk in $1 \times$ TBS for $1 \mathrm{~h}$ at room temperature, the membrane was then incubated overnight at $4^{\circ} \mathrm{C}$ with the primary antibodies against MCP-1, TNF-a, IL-6, IL-10, arginase-1, S1pr1, and S1pr3 , Sphk1, followed by incubation with the appropriate peroxidase-conjugated secondary antibodies for $1 \mathrm{~h}$. Finally, the proteins were detected using an ECL Advanced ${ }^{\mathrm{TM}}$ system. Relative protein levels were calculated by normalization to the amount of $\beta$-actin protein. 
Table 1. Primer sequences for real-time RT-PCR.

\begin{tabular}{llll}
\hline Gene & Accession No & Forward primer $\left(5^{\prime}-3^{\prime}\right)$ & Reverse primer $\left(3^{\prime}-5^{\prime}\right)$ \\
\hline MCP-1 & NM_031530 & TAGCATCCACGTGCTGTCTC & CAGCCGACTCATTGGGATCA \\
TNF- $\alpha$ & NM_012675 & CGTCAGCCGATTTGCCATT & TCCCTCAGGGGTGTCCTTAG \\
IL-6 & NM_012589.1 & AGAGACTTCCAGCCAGTTC & AGTCTCCTCTCCGGACTTGT \\
IL-10 & NM_012854.2 & CTGGCTCAGCACTGCTATGT & GCAGTTATTGTCACCCCGGA \\
Arginase-1 & NM_017134.3 & TGGCGTTGACCTTGTCTTGT & TTTGCTGTGATGCCCCAGAT \\
Sphk1 & NM_001270811.1 & CCCAGGCCTCCATAGTGTTC & GTTTGGGGACGAAGTCGAGA \\
S1pr1 & NM_017301 & TGGCCCCTCCTCAAAGACTA & ACACCACCCACCAAGCTAAA \\
S1pr3 & NM_001271143.1 & TAGCTGAGACTTAGCGGTGG & TGTTGGAACAGGCTCTCGT \\
B-Actin & NM_031144.3 & CACCCGCGAGTACAACCTTC & CCCATACCCACCATCACACC \\
\hline
\end{tabular}

\section{LC-MS/MS assay for S1P quantification}

Kidney tissue samples $(20 \mathrm{mg}$ ) were homogenized in 400 $\mu \mathrm{L}$ of distilled water with electric-homogenizer on ice. Protein assays on each individual homogenate were performed using BCA protein assay kit (KGP150, Nanjing, China) with bovine serum albumin (BSA) as standard. S1P and related compounds were extracted with methanol precipitation. The homogenate was vortex-mixed for $10 \mathrm{~s}$ followed by centrifugation at $12000 \mathrm{r} / \mathrm{min}$ for $5 \mathrm{~min}$. The supernatant was then transferred to clean glass vial and $10 \mu \mathrm{L}$ of the supernatant was injected into the LC-MS/MS system for analysis.

\section{Statistical analysis}

Data are presented as the mean \pm SEM. Data from the three groups were analyzed using one-way ANOVA or the $t$-test. $P$ values $<0.05$ were considered significant.

\section{Results}

Effects of FTY720 treatment on kidney weight and blood biochemical parameters

The kidney weight in the AO rats markedly increased compared with the controls, and FTY720 treatment led to lower kidney weight than the AO group ( $P<0.05$, Table 2$)$. However, the total protein level and the albumin level did not differ among the three groups. Furthermore, serum albumin, serum creatinine and blood urea nitrogen did not change in these groups by the end of the experiment.

\section{Effects of FTY720 on peripheral blood cells}

Peripheral lymphocyte and monocyte counts increased in the AO group compared with the saline control group $(P<0.05)$, and the number of lymphocytes in the FTY720-treated rats was clearly reduced to levels lower than that of the saline control group $(P<0.05$, Figure $1 \mathrm{~A})$. However, FTY720 treatment had no effect on the number of circulating monocytes (Figure 1B).

A

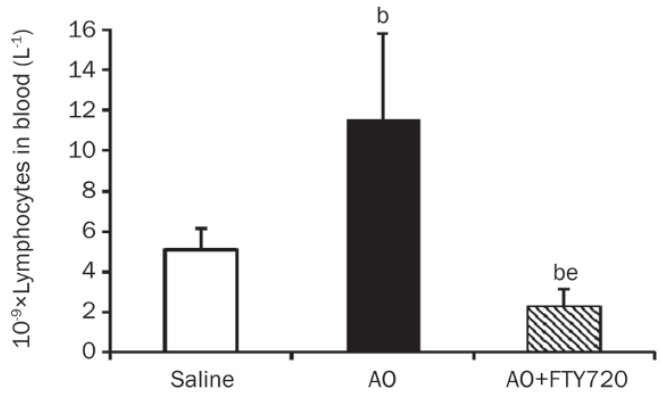

B

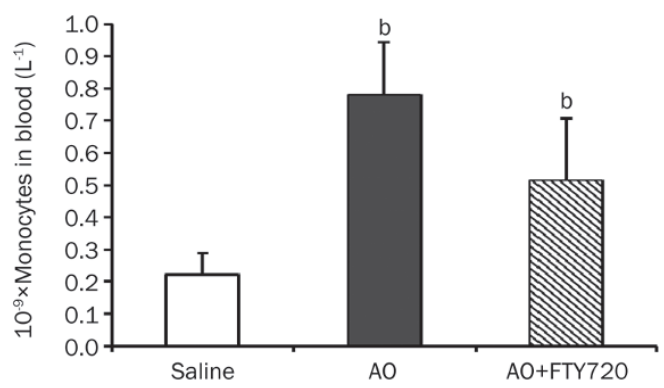

Figure 1. Effect of FTY720 on peripheral blood cells. (A) Lymphocytes in the blood. (B) Monocytes in the blood. Values are the mean \pm SEM. $n=6$. ${ }^{\mathrm{b}} P<0.05$ vs Saline. ${ }^{\mathrm{e}} \mathrm{P}<0.05$ vs AO.

Table 2. Effect of FTY720 on renal parameters in the experimental rats. Values are mean \pm SEM. ${ }^{b} P<0.05$ vs Saline. ${ }^{\text {e }} P<0.05$ vs AO.

\begin{tabular}{lrlrrrr}
\hline Group & $n$ & $\mathrm{KW}(\mathrm{g})$ & $\mathrm{SCr}(\mu \mathrm{mol} / \mathrm{L})$ & $\mathrm{BUN}(\mathrm{mmol} / \mathrm{L})$ & $\mathrm{TP}(\mathrm{g} / \mathrm{L})$ & $\mathrm{Alb}(\mathrm{g} / \mathrm{L})$ \\
\hline Saline & 8 & $2.64 \pm 0.10$ & $8.35 \pm 0.34$ & $46.80 \pm 5.36$ & $56.50 \pm 1.29$ \\
AO & 10 & $4.05 \pm 0.30^{\mathrm{b}}$ & $9.27 \pm 0.47$ & $54.60 \pm 6.11$ & $58.75 \pm 0.96$ & $16.57 \pm 0.97$ \\
AO+FTY720 & 10 & $3.66 \pm 0.32^{\text {be }}$ & $9.02 \pm 0.51$ & $48.20 \pm 8.70$ & $58.6 \pm 5.90$ & $15.11 \pm 2.20$ \\
\hline
\end{tabular}

KW, left kidney weight; SCr, serum creatinine; BUN, blood urea nitrogen; TP, total protein; Alb, albumin. 


\section{Effect of FTY720 on urinary protein and NAG}

After the second week after uninephrectomy or the first week after intraperitoneal BSA injection, the level of proteinuria in the $\mathrm{AO}$ rats progressively increased and reached the highest level at week $7(P<0.05)$. FTY720 treatment significantly reduced proteinuria $(P<0.05$, Figure $2 \mathrm{~A})$. The NAG level in the $\mathrm{AO}$ rats also increased after the second week but was reduced by FTY720 treatment $(P<0.05)$ (Figure $2 \mathrm{~B}$ ).

\section{Effect of FTY720 on histological changes}

The AO rats had marked tubulointerstitial damage characterized by tubular dilation and atrophy, protein casts, inflammatory cell infiltration and renal fibrosis. FTY720 treatment dramatically decreased inflammatory cell infiltration in the tubulointerstitium and reduced tubulointerstitial damage $(P<0.05$, Figure 3).

\section{Effect of FTY720 on tubulointerstitial infiltration with inflamma-} tory cells

The T lymphocyte (CD3+), B lymphocyte (CD20+) and macrophage $(\mathrm{CD} 68+)$ counts in the tubulointerstitium were significantly increased in the $\mathrm{AO}$ rats compared with the saline control group $(P<0.05$, Figure 4$)$. However, these counts were significantly reduced in the FTY720-treated rats $(P<0.05$, Table 3).

\section{Effect of FTY720 on inflammatory cytokine expression}

The renal mRNA and protein expression of chemokines such as monocyte chemoattractant protein-1 (MCP-1) were increased in the $\mathrm{AO}$ rats compared with the saline control group $(P<0.05)$. FTY720 treatment had a greater effect on reducing $\mathrm{MCP}-1$ expression in $\mathrm{AO}$ rats $(P<0.05$, Figure 5$)$.
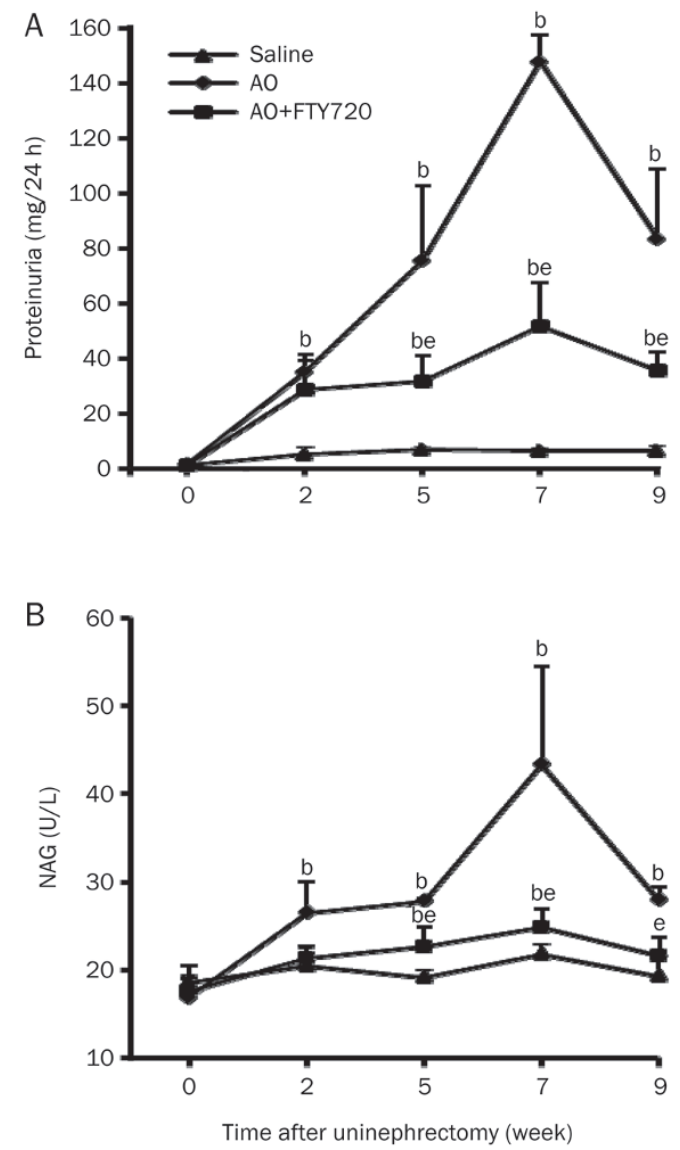

Figure 2. Effect of FTY720 on urinary protein and NAG. Time course of proteinuria (A) and $N$-acetyl- $\beta$ - $D$-glycosaminidase (NAG) activity (B) in saline controls, $A O$ rats, and FTY720-treated rats. Values are presented as the mean \pm SEM. $n=6$. ${ }^{\mathrm{b}} P<0.05$ vs Saline. ${ }^{\mathrm{e}} P<0.05$ vs AO.

A
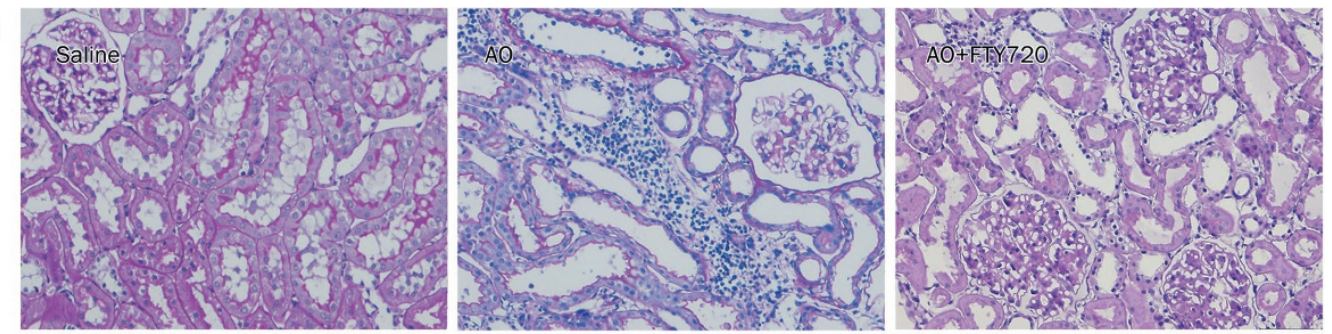

B

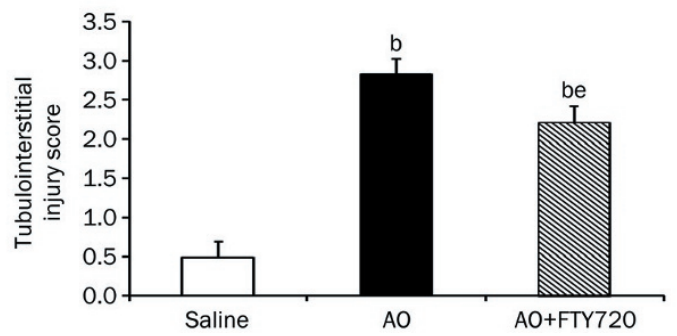

Figure 3. Effect of FTY720 on histological changes in AO rats. (A) PAS stains of tissue as indicated $(\times 200)$. (B) Tubulointerstitial injury score for the three groups. Values are presented as the mean \pm SEM. $n=6 .{ }^{\mathrm{b}} P<0.05$ vs Saline. ${ }^{\mathrm{e}} P<0.05$ vs AO. 
Table 3. Effect of FTY720 on tubulointerstitial inflammatory cells infiltration. Mean \pm SEM. $n=6 .{ }^{\mathrm{b}} P<0.05$ vs Saline. ${ }^{\mathrm{e}} P<0.05$ vs AO.

\begin{tabular}{lccc}
\hline Group & \multicolumn{3}{c}{ Inflammatory cells } \\
& CD3 & CD20 & CD68 \\
\hline Saline & $0.00 \pm 0.00$ & $0.00 \pm 0.00$ & $0.00 \pm 0.00$ \\
AO & $46.30 \pm 9.90^{\text {b }}$ & $56.33 \pm 10.27^{\text {b }}$ & $39.20 \pm 4.78^{\text {b }}$ \\
AO+FTY720 & $16.70 \pm 6.77^{\text {be }}$ & $20.90 \pm 7.29^{\text {be }}$ & $15.00 \pm 2.49^{\text {be }}$
\end{tabular}

Furthermore, the expression of tumor necrosis factor-a (TNFa), interleukin-6 (IL-6), interleukin-10 (IL-10), and arginase-1 was also increased in the $\mathrm{AO}$ rats compared with the saline control group $(P<0.05)$, and the expression of these factors were decreased by FTY720 treatment $(P<0.05$, Figure 5$)$.

\section{Effect of FTY720 on Sphk1 signaling in tubulointerstitial inflam- mation}

Sphingosine kinase 1 (Sphk1) is an enzyme that is involved in generating S1P. Immunohistochemistry analysis showed that Sphk1 was abundantly expressed in the tubulointerstitium of the AO rats. In the FTY720-treated rats, Sphk1 expression was significantly abrogated $(P<0.05$, Figure 6$)$. Furthermore, expression of S1pr1 and S1pr3 were significantly increased compared with the saline control group $(P<0.05)$ and could be inhibited by FTY720 treatment $(P<0.05)$. Consistent with the increases in Sphk1 and S1pr expression, S1P levels in AO rats were markedly increased and reduced by FTY720 treatment $(P<0.05$, Figure 7).

\section{Discussion}

FTY720 \{2-amino-2-[2-(4-octylphenyl) ethyl] propane-1,3-diol\} is a newly developed immunosuppressor isolated from Isaria sinclairi $^{[5]}$. A previous study demonstrated that FTY720 not only acts on $S 1 \mathrm{pr}_{1,3-5}$ but is also a competitive inhibitor of Sphk1 ${ }^{[14]}$. Awad et al showed that FTY720 has anti-inflammatory effects during the early stage of diabetic nephropathy ${ }^{[12]}$. Additionally, Jo et al described its cytoprotective effects in proximal tubular cells during renal ischemia-reperfusion injury $^{[11]}$. More recently, we demonstrated that FTY720 exerts an anti-fibrotic effect in subtotally nephrectomized rats ${ }^{[10]}$. The mechanism by which FTY720 mediates protection in proteinuric nephropathy is not completely understood.

In this study, we demonstrated that FTY720 could inhibit tubulointerstitial inflammation in an $\mathrm{AO}$ rat model of nephropathy, providing a new therapeutic intervention for treating proteinuric nephropathy. Proteinuria is an ominous sign in most patients with nephropathy, and the level of proteinuria correlates with the renal function loss ${ }^{[15]}$. Several mechanisms for proteinuria nephrotoxicity in CKD have been suggested, including direct tubular toxicity, induced synthesis of tubular chemokines, complement activation and hydrogen peroxide generation, leading to inflammatory cell infiltration in the interstitium ${ }^{[2]}$. The $\mathrm{AO}$ rat, as a model of proteinuric nephropathy, has previously shown a high-grade proteinuria

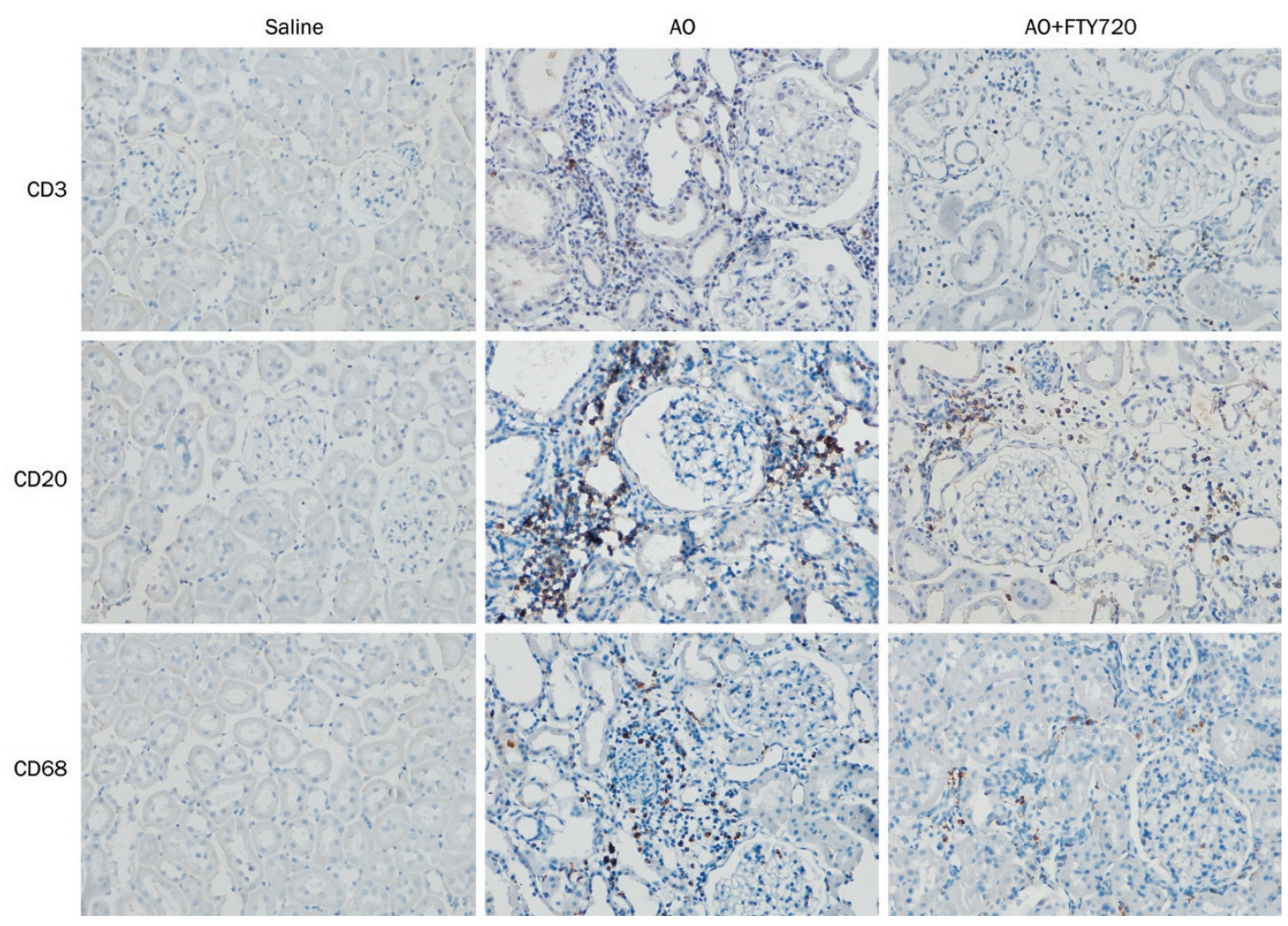

Figure 4. Immunohistochemical staining of lymphocytes and macrophages in three groups. CD3-positive T lymphocytes, CD20-positive B lymphocytes, and CD68-positive macrophages (original magnification is $\times 200$ ). 
A

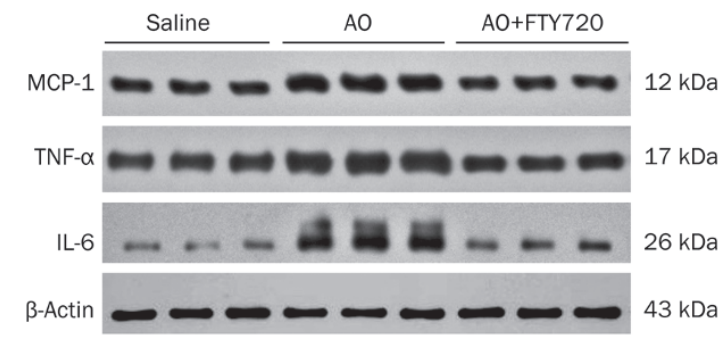

B

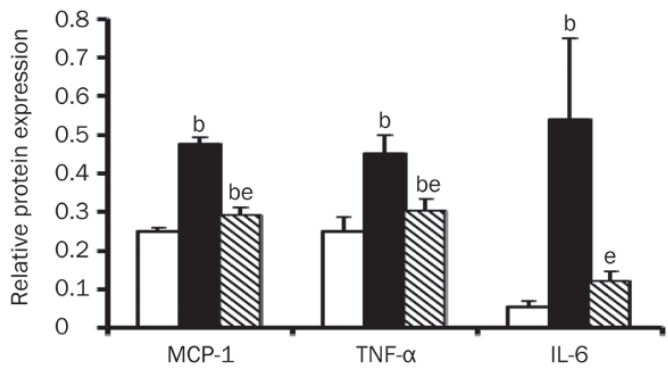

C

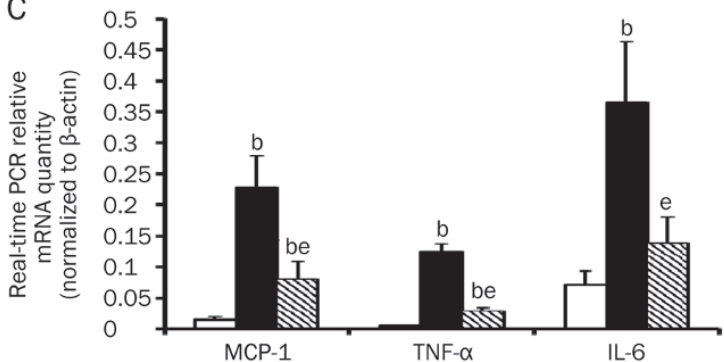

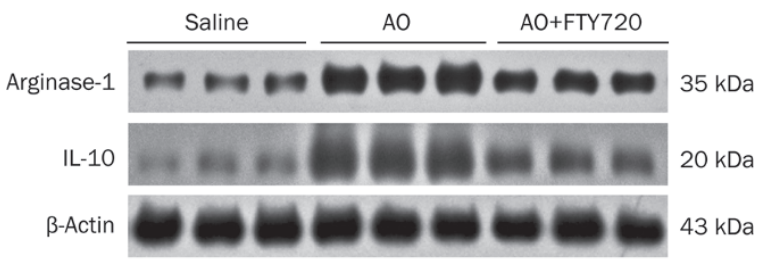
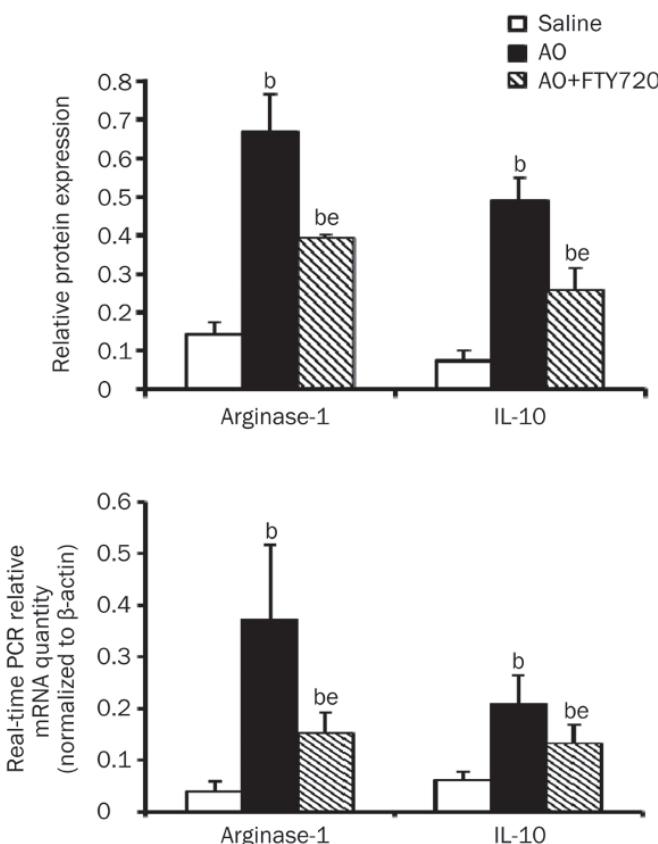

Figure 5. Effect of FTY720 on inflammatory cytokines, including monocyte chemoattractant protein-1 (MCP-1), macrophage-related TNF- $\alpha$ and IL6 , arginase-1 and IL-10, in the three groups. (A) Western blot analysis of MCP-1 and M1 and M2 macrophage-related cytokines; $\beta$-actin was used as control. (B) Quantitative analysis of protein expression of MCP-1 and macrophage-related cytokines. (C) Expression of MCP-1 and macrophage-related cytokines mRNA; $\beta$-actin was used as control. Values are presented as the mean \pm SEM. $n=6 .{ }^{b} P<0.05$ vs Saline. ${ }^{e} P<0.05$ vs AO.

with TI inflammation and subsequent secretion of inflammatory mediators ${ }^{[16]}$. In this study, we found that intraperitoneal injection of BSA significantly increased proteinuria excretion, the number of macrophages and $\mathrm{T}$ and $\mathrm{B}$ lymphocytes in the tubulointerstitium and the expression of associated inflammatory mediators, such as MCP-1, TNF-a, and IL-6.

The severity of tubular and interstitial inflammation is widely accepted to be the driver for developing fibrotic lesions. Interstitial inflammation is characterized primarily by an influx of mononuclear cells, which are composed of monocytes/macrophages and lymphocytes ${ }^{[17]}$. Then, these cells in turn secrete cytokines, which further stimulate resident fibroblasts and tubular epithelial cells to differentiate into matrixproducing cells. Recently, Lee et al suggested that more studies should be focused on renal inflammation rather the later stages of fibrosis ${ }^{[4]}$. Fingolimod (FTY720), a structural analog of sphingosine, was recently approved as a potent immunosuppressor with low cytotoxicity. Once phosphorylated to FTY720-P, FTY720 functions as a modulator of four S1P receptors, resulting in the ubiquitin-mediated degradation of S1P1R and inability of lymphocytes to egress from lymphoid tissues, thus dampening the inflammatory response ${ }^{[18,19]}$. Histological analysis has revealed that the lymph node medullary sinuses of FTY720-treated mice were emptied of lymphocytes, inducing lymphopenia ${ }^{[20]}$. Our current study found that FTY720 treatment induced marked lymphopenia by reducing the number of lymphocytes in circulation and infiltration into the tubulointerstitium of the AO rats. In addition, FTY720 reduced macrophage infiltration in the tubulointerstitium, without any effect on the monocyte count. However, others have demonstrated that FTY720 reduces circulating monocytes over a similar time course as lymphocytes, both in naive and inflammatory conditions in a dose-dependent manner ${ }^{[21]}$.

$\mathrm{S} 1 \mathrm{P}$ is a lipid mediator involved in regulating proliferation, migration, vascular integrity and inflammation in various cell types in the innate- and adaptive-immune systems ${ }^{[22]}$. Sphingosine kinase 1 and 2 (Sphk1 and Sphk2) are the enzymes responsible for producing S1P from sphingosine, and five subtypes of cell surface G-protein-coupled receptors, S1 $\mathrm{pr}_{1-5}$, mediate the action of S1P in various organs. Lymphocytes "sense" an S1P gradient (low concentrations in the lymph nodes versus high concentrations in circulation) with their 
A

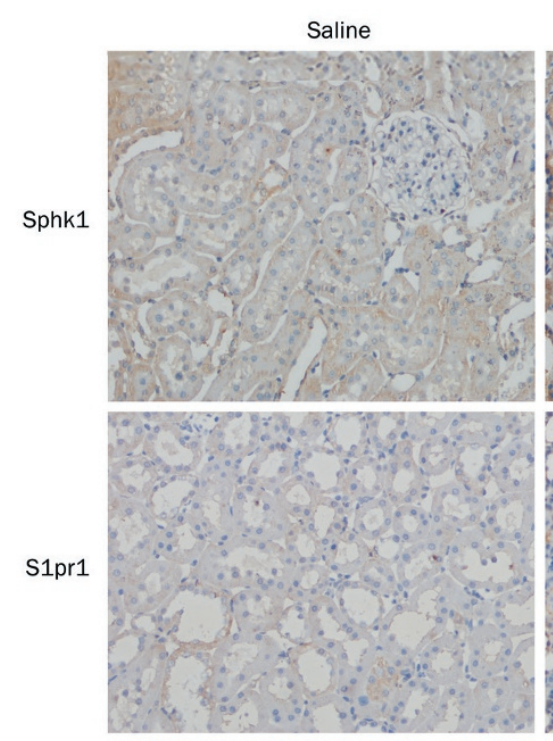

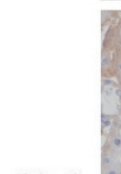

S1pr3
AO
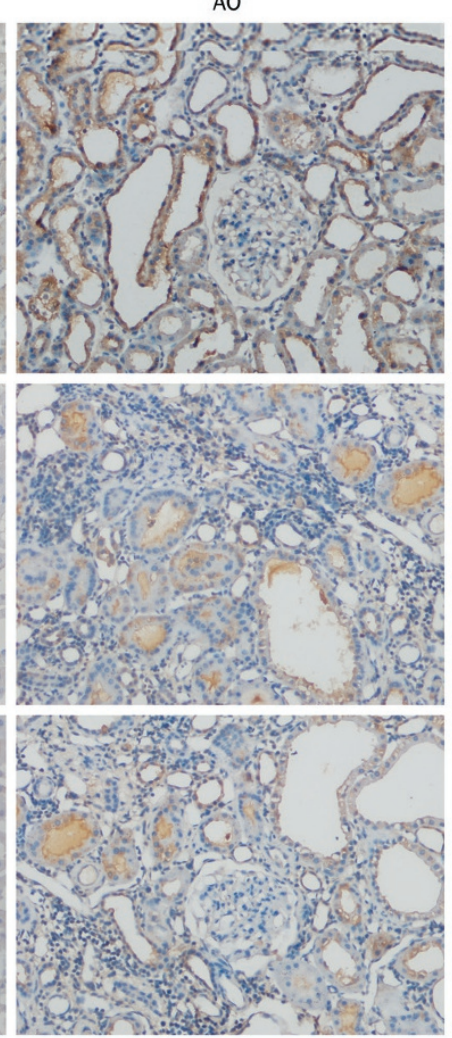
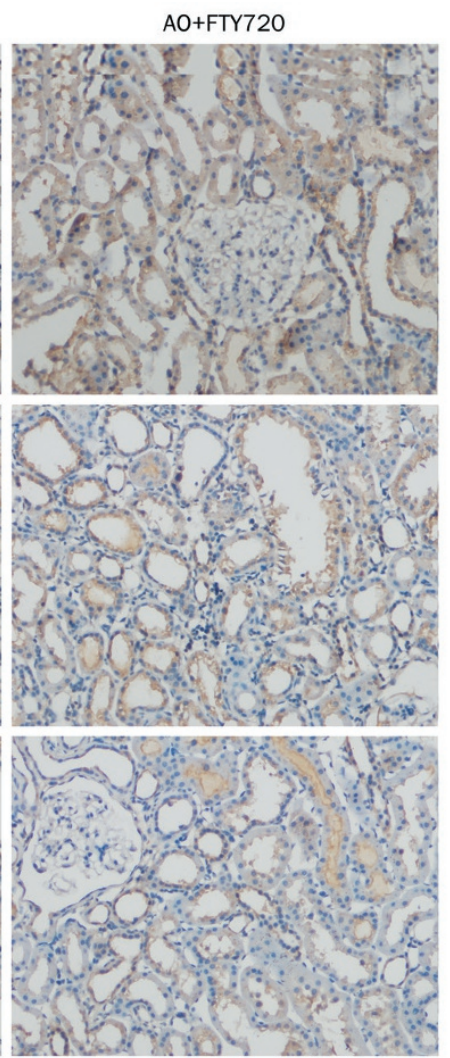
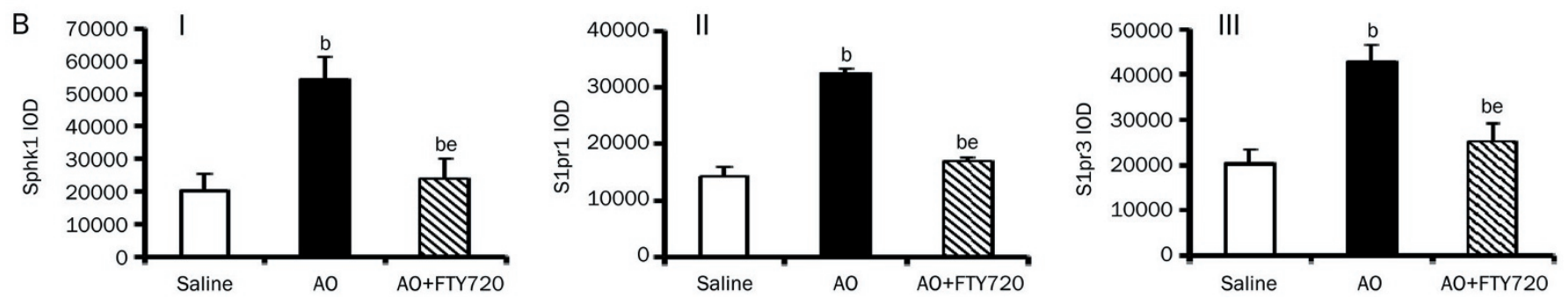

Figure 6. Effect of FTY720 on Sphk1/S1P/S1pr signal-related protein expression in the three groups. (A) Immunohistochemical staining of Sphk1, S1pr1, and S1pr3 in the saline, AO, and AO+FTY720 groups (original magnification is $\times 200$ ). (B) Semiquantitative immunohistochemical analysis of the Sphk1/S1P/S1pr signal-related protein expression in the three groups (I-III). Values are presented as the mean \pm SEM. $n=6$. ${ }^{b} P<0.05$ vs Saline. ${ }^{\mathrm{e}} \mathrm{P}<0.05$ vs AO.

S1P1 receptors, thereby migrating out of the lymphoid organs into the circulation, and this action was found to be blocked by FTY720. In addition, in vivo and in vitro studies have supported the pro-inflammatory role of the Sphk1 pathway in many diseases ${ }^{[23]}$, and various inflammatory signals, including interleukin $1 \beta$, interferon $\gamma$ and lipopolysaccharide (LPS), have been demonstrated to up-regulate Sphk1 expression ${ }^{[24]}$. For example, abundant expression of Sphk1 was found in atherosclerotic lesions in $\mathrm{ApoE}^{-/-}$mice, suggesting a causal role in atherosclerosis by promoting inflammatory monocyte/ macrophage recruitment ${ }^{[3]}$. Additionally, increased activity of the SK/S1P pathway correlates with the initiation and perpetuation of ulcerative colitis (UC) and inflammatory bowel disease (IBD) ${ }^{[23]}$. Moreover, SK1 inhibition has been shown to abrogate underlying inflammatory components, thus decreasing disease parameters ${ }^{[25]}$. Therefore, we examined whether Sphk1/S1P signaling is involved in tubulointerstitial inflammation in proteinuric nephropathy.

Recently, Award et al demonstrated that expression of S1pr1>S1pr3>S1pr2>S1pr4 in mouse kidneys. S1pr5 mRNA level was undetectable ${ }^{[26]}$. Furthermore, both Sphk1 and Sphk2 were shown to be highly expressed in the kidneys ${ }^{[11]}$. Concerning renal diseases, FTY720 has been shown to exert renoprotection in several animal models of renal inflammation, such as models of glomerulonephritis ${ }^{[27]}$, ischemia-reperfusion and diabetic nephropathy. In addition to reducing circulating lymphocytes, other lymphopenia-independent mechanisms for FTY720 have been suggested. FTY720 can down-regulate 
A
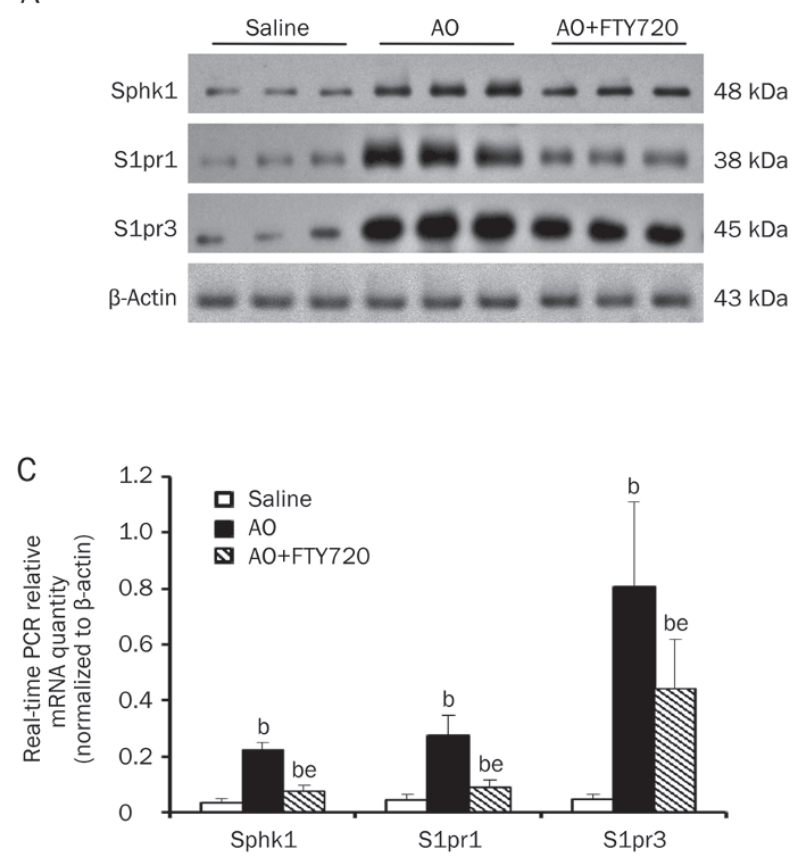
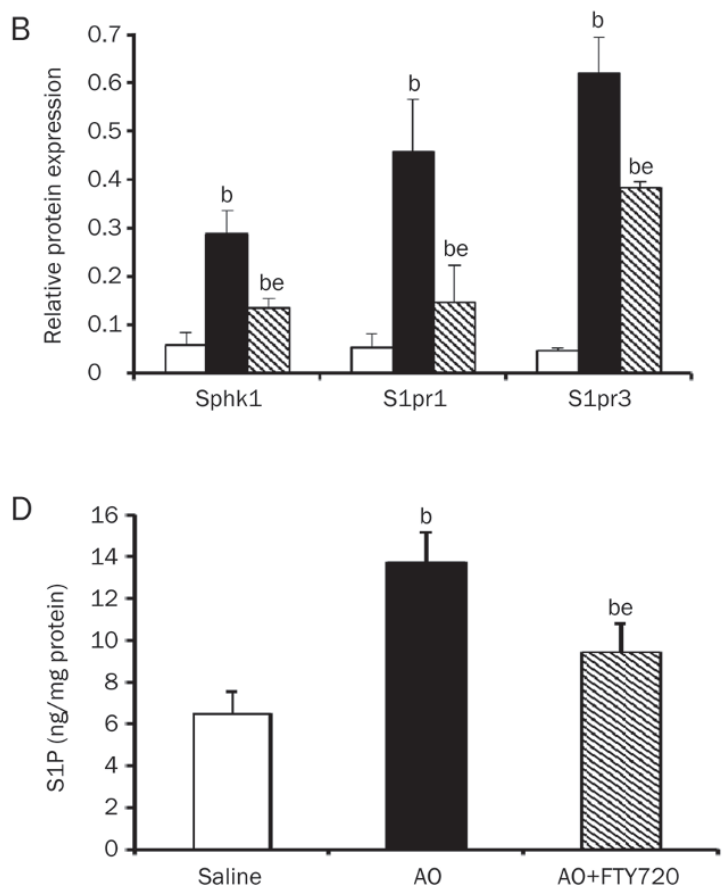

Figure 7. Renal expression of Sphk1/S1P/S1pr related biomarkers in three groups. (A) Western blotting analysis of Sphk1, S1pr1, and S1pr3; $\beta$-actin was used as control. (B) Expression of protein of Sphk1, S1pr1, and S1pr3. (C) Expression of mRNA of Sphk1, S1pr1, and S1pr3; $\beta$-actin was used as control. (D) Sphingosine 1-phosphate (S1P) quantification using LC-MS/MS. Values are presented as the mean \pm SEM. $n=6$. ${ }^{b} P<0.05$ vs Saline. ${ }^{\mathrm{e}} \mathrm{P}<0.05$ vs AO.

the expression of splenic $\mathrm{S}_{1} \mathrm{P}_{1}, \mathrm{~S}_{1} \mathrm{P}_{2}$, and $\mathrm{S}_{\mathrm{P}} \mathrm{P}_{5}$ receptors ${ }^{[28]}$ and TLR 2 and 4 in kidney resident cells ${ }^{[2]]}$, thus reducing the inflammatory process. FTY720 has also been shown to act as a competitive inhibitor of Sphk1. In this study, we found abundant Sphk1 expression in the inflammatory lesions in $\mathrm{AO}$ rats and a high expression of $\mathrm{S}_{1} \mathrm{P}_{1}, \mathrm{~S}_{\mathrm{P}} \mathrm{P}_{3}$ receptors and S1P. FTY720 treatment significantly reduced their expression. The effect was accompanied by inhibition of the inflammatory response in $\mathrm{AO}$ rats, further demonstrating that the anti-inflammatory effects of FTY720 in proteinuric nephropathy could be attributed to suppression of the Sphk1/S1P pathway.

In summary, our study demonstrated for the first time that Sphk1/S1P/S1pr signaling is involved in the tubulointerstitial inflammation induced by albumin overload, and FTY720 exerted an important role in alleviating tubulointerstitial inflammation via the Sphk1 pathway. Additional in vitro experiments are needed to elucidate the exact mechanism.

\section{Acknowledgements}

This study was supported by grants from the Key Project of the National Natural Science Foundation of China (№ 81130010), and Project of Jiangsu Clinical Medical Science (№ BL2014080) to Prof Bi-cheng LIU.

\section{Author contribution}

Min XU designed, performed the research, analyzed the data and wrote the manuscript; Dan LIU and Li-hong DING provided assistance in the research; Kun-ling MA, Lin-li LV,
Hong LIU, and Ri-ning TANG contributed reagents and statistical analyses; Min WU and Yi WEN assisted in the research; Bi-cheng LIU designed the research and reviewed the manuscript.

\section{References}

1 Go AS, Chertow GM, Fan D, McCulloch CE, Hsu CY. Chronic kidney disease and the risks of death, cardiovascular events, and hospitalization. N Engl J Med 2004; 351: 1296-305.

2 Abbate M, Zoja C, Remuzzi G. How does proteinuria cause progressive renal damage? J Am Soc Nephrol 2006; 17: 2974-84.

3 Keul P, Lucke S, von Wnuck Lipinski K, Bode C, Graler M, Heusch G, et al. Sphingosine-1-phosphate receptor 3 promotes recruitment of monocyte/macrophages in inflammation and atherosclerosis. Circ Res 2011; 108: 314-23.

4 Lee SB, Kalluri R. Mechanistic connection between inflammation and fibrosis. Kidney Int Suppl 2010: S22-6.

5 Brinkmann V, Billich A, Baumruker T, Heining P, Schmouder R, Francis G, et al. Fingolimod (FTY720): discovery and development of an oral drug to treat multiple sclerosis. Nat Rev Drug Discov 2010; 9: 88397.

6 Chun J, Brinkmann V. A mechanistically novel, first oral therapy for multiple sclerosis: the development of fingolimod (FTY720, Gilenya). Discov Med 2011; 12: 213-28.

7 Zemann B, Kinzel B, Muller M, Reuschel R, Mechtcheriakova D, Urtz N, et al. Sphingosine kinase type 2 is essential for lymphopenia induced by the immunomodulatory drug FTY720. Blood 2006; 107: 1454-8.

8 Thangada S, Khanna KM, Blaho VA, Oo ML, Im DS, Guo C, et al. Cell-surface residence of sphingosine 1-phosphate receptor 1 on lymphocytes determines lymphocyte egress kinetics. J Exp Med 2010; 
207: $1475-83$.

9 Kveberg L, Bryceson Y, Inngjerdingen M, Rolstad B, Maghazachi AA. Sphingosine 1 phosphate induces the chemotaxis of human natural killer cells. Role for heterotrimeric $\mathrm{G}$ proteins and phosphoinositide 3 kinases. Eur J Immunol 2002; 32: 1856-64.

$10 \mathrm{Ni} \mathrm{HF}$, Chen JF, Zhang MH, Pan MM, Zhang JD, Liu H, et al. FTY720 attenuates tubulointerstitial inflammation and fibrosis in subtotally nephrectomized rats. Ren Fail 2013; 35: 996-1004.

11 Jo SK, Bajwa A, Ye H, Vergis AL, Awad AS, Kharel Y, et al. Divergent roles of sphingosine kinases in kidney ischemia-reperfusion injury. Kidney Int 2009; 75: 167-75.

12 Awad AS, Rouse MD, Khutsishvili K, Huang L, Bolton WK, Lynch KR, et al. Chronic sphingosine 1-phosphate 1 receptor activation attenuates early-stage diabetic nephropathy independent of lymphocytes. Kidney Int 2011; 79: 1090-8.

13 Gadola L, Noboa O, Marquez MN, Rodriguez MJ, Nin N, Boggia J, et al. Calcium citrate ameliorates the progression of chronic renal injury. Kidney Int 2004; 65: 1224-30.

14 Lim KG, Tonelli F, Li Z, Lu X, Bittman R, Pyne S, et al. FTY720 analogues as sphingosine kinase 1 inhibitors: enzyme inhibition kinetics, allosterism, proteasomal degradation, and actin rearrangement in MCF-7 breast cancer cells. J Biol Chem 2011; 286: 1863340.

15 Gorriz JL, Martinez-Castelao A. Proteinuria: detection and role in native renal disease progression. Transplant Rev (Orlando) 2012; 26: 3-13.

16 Ding LH, Liu D, Xu M, Liu H, Wu M, Tang RN, et al. Enalapril inhibits tubulointerstitial inflammation and NLRP3 inflammasome expression in BSA-overload nephropathy of rats. Acta Pharmacol Sin 2014; 35: 1293-301.

17 Muller GA, Markovic-Lipkovski J, Frank J, Rodemann HP. The role of interstitial cells in the progression of renal diseases. J Am Soc Nephrol 1992; 2: S198-205.

18 Cyster JG. Chemokines, sphingosine-1-phosphate, and cell migration in secondary lymphoid organs. Annu Rev Immunol 2005; 23: 127-59.

19 Oo ML, Chang SH, Thangada S, Wu MT, Rezaul K, Blaho V, et al.
Engagement of S1P(1)-degradative mechanisms leads to vascular leak in mice. J Clin Invest 2011; 121: 2290-300.

20 Mandala S, Hajdu R, Bergstrom J, Quackenbush E, Xie J, Milligan J, et al. Alteration of lymphocyte trafficking by sphingosine-1-phosphate receptor agonists. Science 2002; 296: 346-9.

21 Lewis ND, Haxhinasto SA, Anderson SM, Stefanopoulos DE, Fogal $\mathrm{SE}$, Adusumalli $\mathrm{P}$, et al. Circulating monocytes are reduced by sphingosine-1-phosphate receptor modulators independently of S1P3. J Immunol 2013; 190: 3533-40.

22 Rivera J, Proia RL, Olivera A. The alliance of sphingosine-1-phosphate and its receptors in immunity. Nat Rev Immunol 2008; 8: 753-63.

23 Snider AJ, Kawamori T, Bradshaw SG, Orr KA, Gilkeson GS, Hannun YA, et al. A role for sphingosine kinase 1 in dextran sulfate sodiuminduced colitis. FASEB J 2009; 23: 143-52.

24 Snider AJ, Orr Gandy KA, Obeid LM. Sphingosine kinase: Role in regulation of bioactive sphingolipid mediators in inflammation. Biochimie 2010; 92: 707-15.

25 Maines LW, Fitzpatrick LR, French KJ, Zhuang Y, Xia Z, Keller SN, et al. Suppression of ulcerative colitis in mice by orally available inhibitors of sphingosine kinase. Dig Dis Sci 2008; 53: 997-1012.

26 Awad AS, Ye H, Huang L, Li L, Foss FW Jr, Macdonald TL, et al. Selective sphingosine 1-phosphate 1 receptor activation reduces ischemia-reperfusion injury in mouse kidney. Am J Physiol Renal Physiol 2006; 290: F1516-24.

27 Martini S, Kramer S, Loof T, Wang-Rosenke Y, Daig U, Budde K, et al. S1P modulator FTY720 limits matrix expansion in acute anti-thy1 mesangioproliferative glomerulonephritis. Am J Physiol Renal Physiol 2007; 292: F1761-70.

28 Sui M, Zhou J, Xie R, Liu X, Mu S, Jia X, et al. The sphingosine-1phosphate receptor agonist FTY720 prevents the development of anti-glomerular basement membrane glomerulonephritis. Mol Biol Rep 2012; 39: 389-97.

29 Pedregosa JF, Haidar AA, Hirata AE, Franco M, Gomes GN, Bueno V. TLR2 and TLR4 expression after kidney ischemia and reperfusion injury in mice treated with FTY720. Int Immunopharmacol 2011; 11: 1311-8. 